\title{
UMA ABORDAGEM SOBRE A GESTÃO INTEGRADA E PARTICIPATIVA DOS RECURSOS PESQUEIROS COM A CONTRIBUIÇÃO DO MODELO TRADE-OFF
}

\author{
A. C. C. SILVA ${ }^{1}$ e R.T. DANTAS ${ }^{2}$ \\ ${ }^{1,2}$ Instituto Federal do Rio Grande do Norte - Câmpus Natal Central \\ ${ }^{2}$ Universidade Federal de Campina Grande - UFCG \\ adriana.silva@ifrn.edu.br-renilson@dca.ufcg.edu.br
}

Artigo submetido em setembro/2011 e aceito em fevereiro/2013

\section{RESUMO}

A utilização e os modos de apropriação dos recursos naturais de uso comum, em especial os recursos pesqueiros, vêm sendo discutidas na pesquisa acadêmica. Levando isso em consideração, este artigo tem como objetivo fazer uma abordagem sobre a gestão integrada e participativa dos recursos pesqueiros com a contribuição do modelo trade-off adaptado de Policarpo e Santos (2008). Para tanto, foi proposto o estudo do modelo trade-off com o intuito de promover uma reflexão sobre uma proposta de um modelo de gestão integrada para os recursos pesqueiros com a participação dos atores sociais. Buscou-se nas referências bibliográficas o amparo teórico para as discussões em tela. Foi tomado como base os estudos de Policarpo e Santos (2008) que subsidiarão as discussões acerca dos modelos de gestão integrada para os recursos pesqueiros. Como resultado deste estudo, a principal contribuição está concentrada na importância da interação e na participação de atores sociais nas políticas e na tomada de decisão.

PALAVRAS-CHAVE: Gestão integrada, recursos naturais, recursos pesqueiros, pesca artesanal, modelo trade-off.

\section{AN APPROACHN CONCERNING THE INTEGRATED AND PARTICIPATIVE MANAGEMENT OF FISHERIES RESOURCES WITH THE TRADE-OFF TEMPLATE CONTIBUTION}

\section{ABSTRACT}

The use and ways of appropriation of natural resources of common use, remarkably fishery resources, have been discussed at academic surveys. The aim of the present paper is to perform an approach concerning the integrated and participative management of fishery resources with the trade-off template contribution adapted from Policarpo e Santos (2008). Thus, it was proposed the study of the trade-off template in order to promote a reflection on a proposal of an integrated management template for fishery resources with the participation of social actors. The bibliographic references provided the theoretical support for the present discussion. The studies of Polycarp and Santos (2008) were taken as subsidies to discuss the integrated management templates for fishery resources. As a result of this study, the main contribution is focused on the significance of the interaction and participation of social actors in public policy and decision-making processes.

KEY-WORDS: integrated management, natural resources, fisheries resources, handmade fishery, trade-off template. 


\section{UMA ABORDAGEM SOBRE A GESTÃO INTEGRADA E PARTICIPATIVA DOS RECURSOS PESQUEIROS COM A CONTRIBUIÇÃO DO MODELO TRADE-OFF}

\section{INTRODUÇÃO}

Ao longo da história da humanidade, as sociedades buscam incessantemente o desenvolvimento. O que era uma questão de sobrevivência para os primeiros agrupamentos humanos, considerados selvagens ou primitivos, passou a ser tratado como questão filosófica, política, econômica, social e ambiental pelos agrupamentos humanos considerados civilizados, ou com, pelo menos, alto grau de organização. O conceito e as formas de buscar o desenvolvimento evoluíram junto com a ideia de organização e progresso, mas continuam sendo questionados e se tornaram a meta principal das sociedades que buscam uma melhor qualidade de vida presente e futura para seus habitantes. A reflexão sobre o tema desenvolvimento, juntamente com o aumento do impacto ambiental e da importância do conhecimento humano, levou ao crescimento da consciência acerca dos problemas ambientais gerados por padrões de vida incompatíveis com o processo de regeneração do meio ambiente (LIRA, 2008).

A questão ambiental emerge de forma complexa, através de uma problemática contemporânea, no século XX, em virtude da civilização (Revolução Industrial) que se manifestou pela fragmentação do conhecimento e pela degradação do ambiente.

Desde a época da Conferência de Estocolmo, em 1972, as condições de viabilidade de uma modalidade de gestão integrada e descentralizada dos recursos naturais vêm ocupando um espaço cada vez maior no debate sobre desenvolvimento e meio ambiente. Verifica-se, no entanto, que, apesar de os desafios envolvidos na busca de operacionalização dessas ideias serem imensos, necessita-se que os agentes de desenvolvimento aprendam a lidar com o caráter multidimensional e transescalar - no espaço e no tempo - dos usos que se faz da natureza. Além disso, os conflitos de percepção e interesse envolvendo uma grande diversidade de atores sociais - representantes do setor governamental, do setor econômico e da sociedade civil organizadaainda se encontram nos primórdios de uma fase de transição rumo à conquista efetiva da cidadania ambiental no campo da gestão do patrimônio natural e cultural (LIRA, 2008).

Esse enfoque permitiu um novo tipo de análise da gestão dos recursos naturais, apontando o conceito de que os problemas ambientais implicam conflitos de interesses entre segmentos sociais e que, portanto, a gestão pública deve dar margem à identificação desses interesses e dos interessados. Desse modo, a gestão passa a ter não apenas os aspectos técnicos envolvidos em cada questão ambiental, mas também o seu significado econômico, cultural, político e financeiro para todos os segmentos envolvidos (GOLDENSTEIN e SALVADOR, 2005). As dinâmicas de gestão dos recursos pesqueiros no país ilustram bem esse ponto de vista.

Conforme Diegues (1999), as atividades pesqueiras são divididas em três categorias: subsistência, artesanal e industrial (de média e grande escala). A primeira destina-se ao consumo próprio e/ou familiar, enquanto as demais são comercialmente orientadas. As pescarias artesanais são atuantes em toda a costa brasileira e são praticadas por pescadores autônomos, os quais exercem a atividade individualmente ou em parcerias. Essas pescarias empregam apetrechos relativamente simples, e o produto é comercializado, normalmente, através de intermediários. 
A importância do conhecimento ecológico tradicional e do conhecimento local dos pescadores artesanais passou a ser também melhor percebida mediante a disseminação dessa linha inovadora de pesquisa interdisciplinar e orientada para a ação, sendo que o primeiro diz respeito a um conjunto cumulativo de saberes, crenças e práticas geradas por populações tradicionais e transmitidas de geração a geração, sobre as relações dos seres vivos (inclusive humanos) entre si e com o seu meio ambiente (BERKES et al., 2001). Já o conceito de conhecimento local refere-se à generalização progressiva das observações locais feitas por usuários dos recursos naturais em contextos sócio ecológicos específicos, diferindo do conhecimento tradicional pelo fato de não ser produto da transmissão através de várias gerações. Ambos desempenham um papel importante na dinâmica de funcionamento de sistemas comunitários de gestão dos recursos pesqueiros, na medida em que podem ser integrados, pressupondo o cultivo do diálogo de saberes, ao acervo de conhecimentos científicos acumulados e que se ajusta a uma política de empoderamento gradual das comunidades de usuários diretos dos recursos de uso comum (BERKES et al., 2001).

A forma peculiar como a comunidade pesqueira lida com o tempo e suas atividades profissionais ligadas à pesca talvez seja um dos aspectos mais instigantes da vida cotidiana dessas comunidades. Segundo Diegues (1999), a pesca artesanal é caracterizada por ser de pequena escala, com unidade de produção geralmente familiar que, mesmo em dificuldades, continua demonstrando sua viabilidade econômica.

O contato com a realidade das condições de trabalho dos pescadores possibilita o diálogo com camadas sociais que, em face das práticas tradicionais sobre as quais são edificadas, sofrem as transformações advindas de mudanças nos modos de produção e de novas relações sociais que se estabelecem em decorrência dessas mudanças (FONSECA, 2005).

A percepção território-ambiental dos pescadores, construída a partir de suas relações cotidianas com os ambientes de pesca, é um importante instrumento para a gestão dos recursos pesqueiros. É por meio dessas percepções e da relação de coesão entre os pescadores de uma mesma região que eles tiram conclusões acerca da necessidade da criação de regras para o acesso aos recursos disponíveis no território (CRUZ e ALMEIDA, 2010).

Face às coações impostas pelo mercado internacional, num contexto de globalização assimétrica, a organização da pesca industrial vem sendo fortalecida em detrimento das instituições de gestão que operam no nível local ou comunitário, colocando em risco o setor pesqueiro artesanal (DIEGUES, 1995). De acordo com Castello (2007), isso foi evidenciado no período 1945-1995, quando as capturas mundiais marinhas aumentaram quase quatro vezes, de menos de 20 para mais de 80 milhões de toneladas. A partir dos anos 50, assiste-se a um acelerado crescimento e desenvolvimento tecnológico da frota pesqueira e uma expansão de mercado que leva, já nos anos 60, a procurar novos fundos de pesca. 0 desenvolvimento das grandes pescarias de ultramar foi a resposta encontrada pelas potências pesqueiras da época, que fizeram pesados investimentos em embarcações maiores dotadas dos últimos avanços em tecnologia da pesca e navegação. Essa situação levou ao surgimento de inúmeros conflitos sociais, em função da disputa pela utilização dos recursos pesqueiros.

As evidências apontadas na bibliografia disponível indicam que, aproximadamente, $70 \%$ dos estoques considerados mais importantes para a economia pesqueira vêm sendo explorados nas últimas décadas (BERKES et al., 2001).

No Brasil, existem diferentes visões em relação à gestão e propriedade dos recursos pesqueiros. Segundo a legislação vigente, os recursos hídricos e os recursos pesqueiros, são bens 
comuns e, portanto pertencem à União, em acordo com o Código das Águas (ISAAC e CERDEIRA, 2004). Assim, o acesso a esses recursos é livre e, segundo a Lei no. 221/1967, que serve de base para toda legislação pesqueira existente, a pesca pode ser realizada por qualquer pessoa devidamente registrada como pescador profissional (PAIVA, 2004).

Desta perspectiva, no rol dos principais desafios à instituição de novos sistemas de gestão patrimonial de recursos pesqueiros, encontram-se uma série de questões relacionadas à definição e limitação dos usuários e à regulação das modalidades possíveis de apropriação e uso (e.g. tecnologias, volume de capturas), adequando-as às características ecossistêmicas (REBOUÇAS et al., 2006).

Begossi (2004) destaca que, nas políticas pesqueiras e de conservação no Brasil, ainda impera o manejo de caráter centralizador imposto por instituições governamentais. Ressaltando que, uma alternativa à política "de cima para baixo" consiste em envolver as comunidades locais em estratégias de co-manejo, também designado manejo colaborativo, em que os pescadores são peça-chave no processo de decisão e implementação das estratégias de gestão a serem adotadas.

Com isso, faz-se necessário planejar e gerenciar o uso do solo e dos recursos naturais nas regiões das zonas costeiras, pois os ambientes costeiros apresentam elevada fragilidade frente aos processos naturais e intervenções humanas e outras atividades que resultam em pressões ambientais permanentes sobre esses ecossistemas.

Neste sentido, o presente artigo foi desenvolvido com a seguinte composição: introdução, em que será apresentada a importância da gestão dos recursos pesqueiros e a pesca artesanal; na fase seguinte, será abordada a fundamentação teórica, que enfatizará a gestão integrada e participativa dos recursos pesqueiros, a contribuição do modelo trade-off adaptado por Policarpo e Santos (2008) e a utilização do modelo trade-off nos recursos pesqueiros; e, em seguida, as considerações finais.

\section{FUNDAMENTAÇÃO TEÓRICA}

\section{GESTÃO INTEGRADA E PARTICIPATIVA DOS RECURSOS NATURAIS DE USO COMUM}

Nos últimos anos, o debate sobre a gestão dos recursos naturais tem ampliado e atraindo não só o interesse dos pesquisadores, como também dos planejadores e formuladores de políticas de gestão dos recursos naturais. Nesse contexto, segundo Vivacqua e Santos (2008), as regiões costeiras se destacam nesse novo campo de pesquisa, uma vez que são as áreas mais ameaçadas do planeta, justamente por estarem sendo submetidas a uma dinâmica de apropriação e uso desordenados e predatórios do seu imenso potencial em termos de recursos naturais.

A zona costeira, como região de interface entre os ecossistemas terrestres e marinhos, é responsável pela ampla gama de funções ecológicas, tais como a prevenção de inundações, da intrusão salina e da erosão costeira, a proteção contra tempestades, a reciclagem de nutrientes e de substâncias poluidoras e a provisão direta ou indireta de habitats e de recursos para uma variedade de espécies exploradas. A biodiversidade exerce papel fundamental no que se refere a maior parte desses mecanismos reguladores, contribuindo, assim, para a caracterização do conjunto da zona costeira como um recurso finito, resultante de um sistema complexo e sensível 
que envolve uma extraordinária inter-relação de processos e de pressões (VIVACQUA e SANTOS, 2008). A gestão desse recurso é o grande desafio da atualidade.

Devido a isso, os governos têm procurado cada vez mais intervir nas atividades econômicas de seus municípios, com o objetivo de promover o desenvolvimento local sustentável voltado para as necessidades coletivas. Assim, de uma visão de simples articulação de políticas setoriais evoluiu-se, no caso dos municípios, para a compreensão de que uma cidade, ainda que pequena ou média, pode ser vista de maneira ampla como unidade básica de acumulação social, parte onde se articulam as iniciativas econômicas e sociais, culturais e políticas, para gerar uma racionalidade sistêmica (SILVA, 2008).

O desenvolvimento local expressa uma estratégia territorial diante do impacto de fragmentação econômica e sócio-espacial gerada pelas novas redes e fluxos econômicos que surgem nessa transição para um regime de acumulação flexível. Essa dimensão territorial se expressa num caminho de construção social no qual se desenvolvem formas distintas de cooperação. As ameaças para a viabilidade do sistema derivam de alguns fatores principais: a dinâmica da tecnologia, a dinâmica da economia e a dinâmica da população. Todos esses fatores podem levar a uma acelerada taxa de mudanças (BOSSEL, 1999).

$\mathrm{Na}$ prática, os recursos naturais de uso comum tendem a ser controlados mediante combinações desses regimes, existindo variações nas combinações. Há, porém, diferentes interesses em jogo, quando se trata de gerir o que é de todos, dando margem a um debate sobre qual seria o regime de apropriação mais adequado para determinado recurso natural (POLICARPO E SANTOS 2008).

\section{GESTÃO INTEGRADA E PARTICIPATIVA DOS RECURSOS PESQUEIROS}

A administração dos recursos pesqueiros fundamenta-se em estudos sobre os padrões e níveis de explotacão aos quais estão submetidos os estoques de pescado, portanto, a coleta sistemática de informações não é um fim em si mesmo, mas uma etapa indispensável para subsidiar o processo de tomada de decisões políticas, por parte do governo ou do setor produtivo, e deve ser considerada como atividade prioritária (ARAGÃO, 2010).

Marrul Filho (2003) destaca que o regramento da utilização desses recursos é competência do Estado, uma vez que a Constituição Federal estabelece esses recursos como de propriedade pública, principalmente quanto à responsabilidade social, ao dever de proteger o meio ambiente tornando-o sustentável. Embora, verifique-se que o atual modelo de gestão não está sendo demonstrado de forma efetiva, e em diversas ocasiões, o poder público é inoperante com relação às ações ilícitas de determinados grupos de atores sociais. Diante disso, foi desenvolvida uma nova metodologia como forma de alternativa, conhecida como processo de gestão compartilhada de recursos pesqueiros.

Entende-se por gestão compartilhada o compartilhamento de poder e responsabilidades entre o Estado e os usuários dos recursos (tanto os diretos - pescadores, e empresários de pesca, como os indiretos - turistas, consumidores, usuários de recursos hídricos etc.), através do qual se elabora um plano de gestão, que é um conjunto de ações articuladas, com visão de longo prazo e tendo como base um diagnóstico, estabelecendo objetivos, metas, pontos de referência, indicadores, medidas de ordenamento, estruturas de acompanhamento, controle e avaliação (MARRUL FILHO, 2003).

No contexto brasileiro, sistemas de gestão compartilhada que representam arranjos institucionais participativos podem intermediar a proteção das sociedades tradicionais, tais como 
pescadores artesanais e catadores de caranguejos, contra a pressão de práticas insustentáveis de uso dos recursos. O Brasil apresenta em sua legislação recentes avanços quanto à inclusão dos usuários dos recursos que, geralmente, representam parcelas da população menos favorecida, política e economicamente, na gestão dos recursos ambientais. Tais ações refletem-se no reordenamento e na criação de novas políticas públicas nacionais que reconhecem a importância de mecanismos participativos de gestão da pesca e incorporam novos instrumentos, para que todas as partes interessadas participem na formulação e implementação de decisões a respeito dos mesmos (KALIKOSKI et al., 2009).

De acordo com Kalikoski et al. (2009), na prática, inúmeros sistemas de gestão compartilhada são estabelecidos como uma forma mais efetiva para o gerenciamento sustentável dos recursos pesqueiros, sendo implementados ao longo das bacias hidrográficas e da zona costeira brasileira. A forma como se dá a implementação do sistema de gestão compartilhada na pesca diferencia-se, a depender do grau de participação efetiva das comunidades e do grau de devolução do poder do governo para as comunidades locais no processo de tomada de decisão.

A gestão compartilhada dos recursos pesqueiros é definida como uma situação em que as instâncias governamentais legalmente responsáveis por essa gestão e a comunidade de usuários diretos do recurso compartilham a responsabilidade desse manejo. É uma situação que está sendo observada em vários níveis de complexidade e em diferentes contextos socioambientais por todo o continente sul-americano (BEGOSSI, 2004). Nesse contexto, no âmbito específico da gestão compartilhada de recursos pesqueiros, verificamos os chamados Acordos de pesca (ISAAC e CERDEIRA, 2004).

Acordos de pesca são instrumentos de gestão coletiva dos recursos pesqueiros (podendo se estender a outros recursos naturais) que, através do diálogo entre os pescadores e os órgãos responsáveis pela legalização e fiscalização da atividade pesqueira, estabelecem normas de apropriação desses recursos por meio de portarias que possuem a força de leis através de Instruções Normativas (RASEIRA, 2007). Segundo Cruz e Almeida (2010), tais acordos têm sido feitos com objetivo de reduzir o esforço de pesca e aumentar sua produtividade. A partir dos Acordos de pesca, os pescadores adquirem a responsabilidade de gerir os recursos pesqueiros que estão disponíveis no território, juntamente com os órgãos responsáveis pela fiscalização e legalização da atividade na localidade, através de um processo de co-gestão dos recursos pesqueiros (gestão compartilhada). A participação dos pescadores na construção dos Acordos de Pesca faz com que estes se identifiquem com as regras criadas, gerando um laço de identidade com esses acordos, o que facilita seu processo de monitoramento e cumprimento . Dessa forma, os acordos de pesca são uma forma de democratização da gestão dos recursos pesqueiros, que fortalecem os laços territoriais dos grupos de pescadores que exercem suas atividades em determinados rios, lagos e igarapés devido ao poder de gestão desses recursos serem compartilhado entre esses pecadores e os órgãos responsáveis pela fiscalização e legalização da atividade, incidindo diretamente na construção de territorialidades coletivas no monitoramento de um bem comum: os territórios aquáticos (CRUZ e ALMEIDA 2010).

Situações de gestão compartilhada do recurso pesqueiro estão geralmente associadas ao uso coletivo desse recurso por um grupo organizado, cujas regras de utilização são estabelecidas visando o controle e conservação do recurso (D'ARRIGO, 2010). Essas regras são fortemente baseadas no conhecimento regional dessas comunidades referente à dinâmica dos ecossistemas e das suas espécies associadas (BEGOSSI, 2004). 
De acordo com Bouamrane e Antona (1998), as especificidades de cada contexto social, econômico e cultural não são levadas em consideração. Mesmo assim, é consenso que há diversos instrumentos de apoio de tomada de decisão para sistemas de gestão de recursos naturais de uso comum, ou seja, instrumentos que auxiliam nas escolhas dos atores sociais entre diferentes alternativas possíveis.

Considerando a especificidade do sistema de gestão dos recursos pesqueiros, é importante destacar a contribuição do modelo trade-off para auxiliar nas escolhas dos atores sociais individuais e/ou coletivos para a tomada de decisão. Pois, segundo Policarpo e Santos (2008), a maioria dos instrumentos - convencionais - identifica o problema, estabelece os possíveis cenários e seleciona esse cenário, de acordo com as preferências dos tomadores de decisão.

Portanto, será abordada a contribuição do modelo trade-off aplicado por Policarpo e Santos (2008) na gestão dos recursos pesqueiros.

\section{A CONTRIBUIÇÃO DO MODELO TRADE-OFF NA GESTÃO DOS RECURSOS PESQUEIROS MODELO TRADE-OFF}

Na percepção de Brown et al. (2002), a análise trade-off é de grande importância como instrumento na promoção de uma gestão participativa e integrada. De acordo com os autores, esse modelo consiste em harmonizar diversos usos dos recursos, com base em representações e seus possíveis impactos, permitindo a identificação dos diversos atores sociais, seus diferentes interesses, visões, conhecimentos e valores envolvidos no sistema de gestão e, assim, modificálo. É uma análise flexível que pode ser modificada de acordo com o desenvolvimento das preferências dos atores sociais ou quando uma nova informação científica ou social se torna disponível, além de poder ser aplicada interativamente ao longo do tempo.

O processo de análise de trade-off, através da combinação de processos deliberativos, avaliação de impactos e construção de consenso, possibilita que a deliberação e a análise possam coexistir e contribuir para as tomadas de decisão no sistema de gestão (POLICARPO e SANTOS, 2008).

De acordo com Policarpo e Santos (2008), o modelo trade-off pode ser detalhado em sete etapas:

1) Identificar os atores sociais envolvidos no sistema de gestão: verificar se os atores sociais são indivíduos ou grupos que manifestam algum tipo de interesse ou alguma reivindicação no processo de apropriação e gestão de recursos naturais.

2) Categorizar dos atores sociais em grupos prioritário: Identificar os atores que estão engajados ativamente no processo de gestão e verificar a influência do nível de poder que os atores sociais têm sobre o resultado de uma decisão e de importância.

3) Explorar os conflitos: verificar a oposição de valores ou necessidades. A falta de informação, de incompatibilidade de valores e crenças de ordem religiosa e/ou cultural, de relações pode gerar conflitos quando afetam outros usuários. E entendendo quem são os atores sociais e quais são seus interesses, é possível explorar seus conflitos.

4) Analisar quem deve ser incluído ou excluído da deliberação: a partir das seguintes etapas: Primários - são os incluídos. Devem sempre manter-se interessados, comprometidos $e$ participantes no processo; Secundários - são os Consultados - incluem gestores dos recursos e responsáveis pelas decisões, mas devem evitar encontrar-se com os primários; Externos - são os 
informados - sua presença pode inibir a discussão e colocar em risco a possibilidade de todos os atores sociais trabalharem juntos em um estágio posterior.

5) Utilizar de técnicas de engajamento: incluir os atores sociais nos processos deliberativos através de apresentações públicas, encontros abertos, grupo focal, entrevistas individuais e semiestruturadas, pesquisa informal, questionário. Assim como o diálogo participativo, com discussões e deliberações e a aplicação de um modelo de co-gestão.

6) Entender as preferências dos atores sociais: incluir os atores sociais nos processos de tomada de decisão e entender suas diferentes preferências, assim como a influência de seu comportamento e o ambiente em que estão inseridos.

7) Gerar informações pertinentes sobre impactos de cenários: informar sobre os impactos dos possiveis cenários objetivando minimizar os conflitos e a distribuição equitativa, eficiência e efetividade e, principalmente, a legitimidade e a confiança produzidas pelos atores sociais.

A partir das etapas do modelo trade-off adaptado por Policarpo e Santos (2008), percebese a maneira de como organizar o jogo de atores sociais individuais e/ou coletivos envolvidos no processo, levando em consideração os diversos interesses, muitas vezes conflitantes, dos diversos usuários do recurso. Uma parte importante dessa análise a ser aplicada na gestão dos recursos pesqueiros é viabilizar um cenário que possa ocorrer a participação dos atores sociais que utilizam, direta ou indiretamente, esse recurso. Assim, é necessário construir o seguinte cenário: 1) Moradores que façam parte atualmente de uma organização que representem os interesses das comunidades locais, 2) Moradores que não estejam envolvidos e nem acompanhando as atividades de gestão, 3) Proprietários cujas atividades e interesses não necessariamente estejam relacionados aos objetivos da gestão, 4) Moradores do entorno, 5) Responsáveis pela gestão, 6) ONGs envolvidas, 7) Autoridades municipais locais, 8) Instituto de pesquisas.

Portanto, o modelo trade-off, aplicado por Policarpo e Santos (2008), proposto neste artigo, teve como objetivo fazer uma abordagem sobre a gestão integrada e participativa dos recursos pesqueiros, identificando os atores sociais, individuais e/ou coletivos, e capacitá-los no processo da gestão dos recursos pesqueiros.

\section{CONSIDERAÇÕES FINAIS}

A utilização sustentável de recursos naturais, especialmente de recursos pesqueiros, é um grande desafio que precisa ser desenvolvido a partir de seis etapas: política, econômica, social, cultural, ambiental e técnica. Os recursos pesqueiros podem ser utilizados economicamente pela pesca industrial, assim como pela comunidade tradicional, utilizando a pesca como subsistência.

É notório ressaltar que, para promover o uso sustentável desse recurso, destacam-se diversos aspectos envolvidos no processo. O primeiro é o técnico-científico, por meio do qual se tem informações sobre os aspectos biológicos das espécies de uso econômico, assim como sobre as interações com as outras espécies, realizando estatísticas de pesca e a avaliação de estoques, com o intuito de conhecer o potencial e utilizar em uma administração sustentável. O segundo é a questão administrativa, que definirá as medidas de ordenamento, tais como, tamanho mínimo de captura, número de pescadores profissionais, quantidade de captura e o período de proibição de pesca. O terceiro é a diretriz política, que determinará a conciliação dos diferentes usos de terra, mantendo a integridade dos recursos naturais. O quarto é o econômico-social, em que, na grande maioria, os pescadores apresentam baixa escolaridade e pouca capacidade de 
organização e são responsabilizados pela falta ou pela diminuição de peixes, principalmente pela incapacidade em dá resposta à sociedade.

Tomando como base a contribuição do modelo trade-off, adaptado por Policarpo e Santos (2008), abordado neste artigo, constatou-se que uma das formas de discussão e de democratização das decisões quanto ao uso sustentável e manutenção da atividade pesqueira é a existência de um órgão colegiado, onde os conflitos de interesse possam ser resolvidos de forma integrada e participativa. Nesses fóruns, as partes envolvidas e interessadas podem ser ouvidas e, baseando-se em argumentos técnico-científicos, aplicar um processo de negociação com o intuito de alcançar um consenso que propicie a continuidade de uso sustentável dos recursos pesqueiros, de forma que a tomada de decisão concilie os aspectos econômicos, sociais e ambientais.

Nesse contexto, para que a atividade pesqueira seja sustentável, trazendo benefícios a esse recurso, faz-se necessário que os instrumentos existentes possam ser utilizados de forma lógica e racional, considerando as informações técnico-científicas, os instrumentos de manejo da pesca e o uso adequado do ambiente que assegure as condições de existência e sobrevivência dos peixes. A proibição da pesca não garantirá o retorno do peixe, e sim através da gestão integrada e participativa que contemple os diferentes interesses de todos os segmentos da sociedade envolvidos nesse tema. Isso implicará na intervenção direta na produção de conhecimento da realidade, no planejamento, na execução, no controle, na avaliação e no redimensionamento das ações a partir das demandas locais.

Diante desse contexto, somente se dará de forma efetiva se a participação da sociedade estiver organizada e legitimamente representada por suas organizações nas instâncias de tomada de decisões.

\section{REFERÊNCIAS}

ARAGÃO, M. C. O.; CURADO, F. F.; SOUZA, R. M. E. Relações socioambientais na comunidade pesqueira Mem de Sá, Sergipe. In: ENCONTRO NACIONAL DA ASSOCIAÇÃO NACIONAL DE PÓSGRADUAÇÃO E PESQUISA EM AMBIENTE E SOCIEDADE, 5., 2010, Anais..., Florianópolis, 2010. p. 01-13.

BEGOSSI, A. Ecologia Humana. In: BEGOSSI, A. (org.) Ecologia de Pescadores da Mata Atlântica e da Amazônia. São Paulo: Fapesp/Hucitec, 2004. 332p.

BERKES, F.; MAHON, R.; MCCONNEY, P.; POLLNAC, R. C.; POMEROY, R. S. Managing smallscale fisheries: alternative directions and methods. International Development Research Centre, Ottawa, 2001.

BOSSEL, H. Indicators for sustainable development: theory, method, application: a report to the Balaton Group. Winnipeg: IISD, 1999. 138p. ISBN:1-895536-13-8.

BOUAMRANE, M.; ANTONA, M. Do ecodesenvolvimento à gestão viável de recursos renováveis: o exemplo das agro-florestas na Indonésia. In: P. F. Vieira, M. A. Ribeiro, R. M. Franco e R. C. Cordeiro (Orgs.). Desenvolvimento e meio ambiente no Brasil: a contribuição de Ignacy Sachs. Porto Alegre: Pallotti; Florianópolis, 1998.

BROWN, K.; TOMPKINS, E. L. ADEGER, W.N. Making Waves: integrating coastal conservation and development. Earthscan. 2. ed., 2002. 
CASTELLO, J.P. Gestão sustentável dos recursos pesqueiros, isto é realmente possível? PanAmerican Journal of Aquatic Sciences, v. 2 , n.1, p. 47-52, 2007.

CRUZ, M. N.; ALMEIDA, O. T. Estratégias para a co-gestão dos recursos pesqueiros no estuário amazônico: o caso dos acordos de pesca em Abaetetuba-PA. In: SIMPÓSIO INTERNACIONAL DE GEOGRAFIA TERRITÓRIO E PODER, 1., 2010, Anais..., Curitiba, 2010.

D'ARRIGO,R.C.P; MOTA, S.; CÂMARA, E. O. O Processo participativo na gestão dos recursos pesqueiros na bacia amazônica - Casos de pactos sociais formalizados no contexto da Amazônia legal brasileira - Acordos de Pesca. In: SEMINÁRIO ÁREAS PROTEGIDAS E INCLUSÃO SOCIAL, 2, 2006. Anais..., Rio de Janeiro. 2006. CD-ROOM.

DIEGUES, A. C. A pesca artesanal no litoral brasileiro: cenários e estratégias para sua sobrevivência. Pescadores artesanais - entre o passado e o futuro. FASE, n. 38, 74 p., 1988.

DIEGUES,A.C. Povos e mares: leituras em sócio-antropologia marítima. São Paulo: NUPAUB-USP, 1995.

DIEGUES, A. C. A sócio-antropologia das comunidades de pescadores marítimos no Brasil. v. 3, p. 361-373, 1999.

FONSECA, A. C. M. Histórias de pescador: as culturas populares nas redes das narrativas. Tese (Doutorado em Letras). 2005. 370f. Universidade Federal da Paraíba João Pessoa, 2005.

GOLDENSTEIN, S.; SALVADOR, Z. Sustentabilidade e da gestão da água e desenvolvimento sustentável. In: DOWBOR, LADISLAU E TAGNIN, RENATO. Administrando a água como se fosse importante. - São Paulo: Editora Senac, São Paulo, 2005.

ISAAC, V. J. ; CERDEIRA, R. G. P. Avaliação e monitoramento de impacto dos acordos de pesca na região do médio Amazonas. In: Documentos Técnicos 3. Manaus: Ibama/Provarzea. 2004.

KALIKOSKI, D. C.; SEIXAS, C. S.; ALMUDI, T. Gestão compartilhada e comunitária da pesca no Brasil: avanços e desafios. Ambiente \& Sociedade, Campinas, v. 12, n.1, p. 151-172, jan.-jun. 2009.

LIRA, W. S. Sistema de Gestão do conhecimento para indicadores de sustentabilidade - SIGECIS: Proposta de uma metodologia. 2008. 176f. Tese (Doutorado em Recursos Naturais) Universidade Federal de Campina Grande, Campina Grande. 2008.

MARRUL-FILHO, S. Crise e sustentabilidade no uso dos recursos pesqueiros. Brasília: IBAMA, 2003.

PAIVA, M. P. Administração Pesqueira no Brasil. Rio de Janeiro: Interciência, 2004.

POLICARPO, M. A.; SANTOS, C. R. Proposta metodológica de uma gestão integrada e participativa dos recursos naturais de uso comum: a contribuição da análise trade-off. Revista de estudos ambientais, v.10, n. 2, p. 71-87, jul./dez. 2008.

RASEIRA, M. B. Monitoramento participativo dos acordos de pesca: como avaliar a captura de pescado Disponível em: <http://www.sepaq.pa.gov.br/files/u1/diag_pesca2008.html.> Acesso em 05 mai 2011.

REBOUÇAS, G. N. M.; FILARDI, A. C. L.; VIEIRA, P. F. Gestão integrada e participativa da pesca artesanal: potencialidades e obstáculos no litoral do estado de Santa Catarina. Ambiente \& Sociedade, v. 9, n.2, jul./dez. 2006. CD-ROOM. 
SILVA, M. G. Sistema de Indicadores para viabilização do desenvolvimento local e sustentável: uma proposta de modelo de sistematização. 2008. 238f. Tese (Doutorado em Recursos Naturais) - Universidade Federal de Campina Grande, Campina Grande, 2008.

VIVACQUA, M.; SANTOS, C. R. Desenvolvimento Territorial Sustentável: os desafios para a Gestão integrada das Unidades de Conservação Costeiras. Disponível em: < http://br.monografias.com/trabalhos917/territorial-sustentavel-costeiras/territorial-sustentavelcosteiras.shtml.> Acesso em: 21 mar.2010. 